\title{
The effects of stereo shift angle, geometric magnification and display zoom on depth measurements in digital stereomammography
}

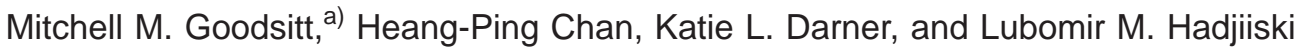 \\ Department of Radiology, University of Michigan, Ann Arbor, Michigan 48109-0030
}

(Received 23 January 2002; accepted for publication 15 August 2002; published 28 October 2002)

\begin{abstract}
We are developing virtual three-dimensional (3-D) cursors for measuring depths in digital stereomammograms. We performed a study to investigate the effects of stereo shift angle, geometric magnification, and display zoom on the accuracy of depth measurements made with a virtual 3-D cursor. A phantom containing 50 low contrast fibrils at depths ranging from 1 to $11 \mathrm{~mm}$ was imaged with a full-field digital mammography system. Left- and right-eye images were generated at stereo shift angles of $\pm 3^{\circ}$ and $\pm 6^{\circ}$, using either contact or $1.8 \times$ geometric magnification geometry. The images were viewed on a high-resolution stereoscopic display system in normal and $2 \times$ zoom mode. Observers viewed the images with stereo glasses and adjusted the depth of a cross-shaped virtual cursor to best match the perceived depth of each fibril. The results for two trained observers with excellent stereo acuity were nearly identical when viewing the same images. The average root mean square errors for the two observers were $1.2 \mathrm{~mm}\left( \pm 3^{\circ}\right.$ contact, no zoom), $1.3 \mathrm{~mm}\left( \pm 3^{\circ}\right.$ contact zoom), $0.8 \mathrm{~mm}\left( \pm 6^{\circ}\right.$ contact, no zoom), $0.6 \mathrm{~mm}$ ( $\pm 6^{\circ}$ contact, zoom), $0.8 \mathrm{~mm}( \pm 3$ magnification, no zoom), $0.7 \mathrm{~mm}\left( \pm 3^{\circ}\right.$ magnification, zoom), and $0.2 \mathrm{~mm}\left( \pm 6^{\circ}\right.$ magnification, no zoom). One observer repeated the entire study for two additional fibril phantom configurations. Combining all the results, we found that for the contact geometry increasing the stereo shift angle from $\pm 3^{\circ}$ to $\pm 6^{\circ}$ improved the depth measurement accuracy by factors of about 1.2-4.0. Zooming did not provide observable improvement in the depth measurement accuracy; sometimes having no effect, sometimes improving the accuracy, and other times reducing the accuracy, with no general trends. Its effect is likely within experimental errors. However, the stereo effect was more readily visualized in the zoom mode. Geometric magnification improved the depth measurement accuracy. The best accuracy among all cases was about $0.2 \mathrm{~mm}$, obtained with geometric magnification using a stereo angle of $\pm 6^{\circ}$. This is the mode we recommend for obtaining accurate depth measurements with virtual cursors in stereomammograms. (C) 2002 American Association of Physicists in Medicine. [DOI: 10.1118/1.1517615]
\end{abstract}

Key words: stereomammography, stereoscopic, virtual cursor, 3-D imaging

\section{INTRODUCTION}

Tissue superposition makes it difficult to accurately interpret conventional mammograms. Such mammograms are acquired using a single projection method whereby (ignoring scatter) the density at a point in the image represents the summation of the attenuation of all tissues along a ray extending from the $\mathrm{x}$-ray tube focal spot to that point. The superpositon of tissues along the rays decreases image contrast and can result in the camouflaging of masses and microcalcifications within dense tissue. It can also lead to superimposed structures having mass-like appearances. The superpositon problem can be reduced or eliminated by generating and viewing 3-D mammograms via multiprojection techniques such as stereoradiography, ${ }^{1-7}$ tomosynthesis, ${ }^{8-10}$ and computed tomography. ${ }^{11,12}$

We have been investigating digital stereomammography. This is a computerized version of an analog technique that was first described by Warren in $1930 .{ }^{13}$ Both the new and old techniques involve taking two separate mammograms, one with the $\mathrm{x}$-ray tube at a positive angle (e.g., $+3^{\circ}$ ) relative to a normal to the detector and the other with the $\mathrm{x}$-ray tube at an equal but opposite angle (e.g., $-3^{\circ}$ ) about the normal. One of the images is viewed with the left eye and the other with the right eye. Our brain fuses the images together to create a 3-D effect. The old technique required taking two films in roughly the same projection. As such, it had several disadvantages, including at least twice the $\mathrm{x}$-ray dose, film cost, and processing time. It also required increased procedure time and radiologist viewing time. Radiologists, in general, eventually decided that these disadvantages outweighed the 3-D visualization advantage, and film stereomammography was discontinued. Digital mammography eliminates or reduces most of these advantages, thereby making digital stereomammography a potentially viable technique. In contrast to screen-film systems, which have sigmoid-shaped response curves, digital detectors have a linear response. Thus, the response curve of the digital detector does not degrade image contrast at lower doses, and it may be possible to utilize half the normal dose for each digital image. The two images of the stereo image pair will be integrated by the obsever's eye-brain system to yield about the same signalto-noise ratio as in a single image taken with the same total dose. ${ }^{14}$ With digital systems, image processing and display are almost instantaneous. Also, the method of examining the 
images, displayed on a television monitor and viewed with liquid crystal display (LCD) glasses that are synchronized so the left eye sees one image and the right the other, is more convenient and less time consuming than the film counterpart.

We have been investigating the use of virtual 3-D cursors for measuring depths in digital stereomammograms. ${ }^{3,5,6}$ Leduc et al. ${ }^{1}$ have also performed research in this area. The 3-D cursors are generated with computer graphics and are overlaid on the digital mammograms. In our initial studies reported previously, ${ }^{3}$ we determined the accuracies of observers' measurements of the depths of horizontally and vertically oriented nylon filaments that simulate fibrils in mammograms. We found that when observers used a cross-shaped cursor, they could determine depths of vertically oriented fibrils with accuracies [root mean square (rms) errors] of $0.4-1.3 \mathrm{~mm}$, but their accuracies were degraded for horizontally oriented fibrils (rms errors of 1.9-4.2 mm). In a subsequent study, ${ }^{5}$ we found that use of a comb-shaped cursor improved the accuracies (reduced the rms errors) of observers' depth measurements of the horizontally oriented fibrils by $0.1-1.4 \mathrm{~mm}$. With this cursor, two of the observers were able to measure the absolute depths of the horizontal fibrils with much improved accuracies of $0.8-1.0 \mathrm{~mm}$. The images for our previous studies were generated with a Fischer (Denver, CO) MammoVision Sterotaxic unit, using a stereo shift of $\pm 2.5^{\circ}$. More recently, our Radiology department obtained a GE (Milwaukee, WI) Senographe 2000D full-field digital mammography system. In this report, we describe a study that was performed using images acquired with this system. We investigated the effects to stereo shift angle, geometric magnification, and display zoom on the accuracy of depth measurements made with a virtual 3-D cursor.

\section{MATERIALS AND METHODS}

\section{A. Phantom}

We employed the same multi-layered fibril phantom that was used in our previous studies. ${ }^{3,5,6}$ This phantom consists of six $1 \mathrm{~mm}$ thick Lexan sheets each separated by $1 \mathrm{~mm}$ spacers. A $5 \times 5$ matrix of $8 \mathrm{~mm}$ long, $0.53 \mathrm{~mm}$ diameter nylon fibrils is placed on the plates with 25 fibrils oriented vertically (perpendicular) and 25 horizontally (parallel) relative to the stereo shift direction. The depths and orientations of the fibrils were randomized and organized such that one horizontal fibril crossed one vertical fibril at each of the 25 matrix positions. The order of the Lexan layers could be changed to create many independent phantom configurations. For our present experiments, we rotated the phantom $45^{\circ}$. This resulted in 25 of the fibrils being oriented at $+45^{\circ}$ and 25 at $-45^{\circ}$ relative to the stereo shift direction. Thus, all fibrils had both horizontal and vertical components.

\section{B. Stereo image acquisition}

As mentioned above, the images were generated with a GE Senographe 2000D digital mammography system. The pixel size for this system is $100 \mu$ in contrast to the $50 \mu$

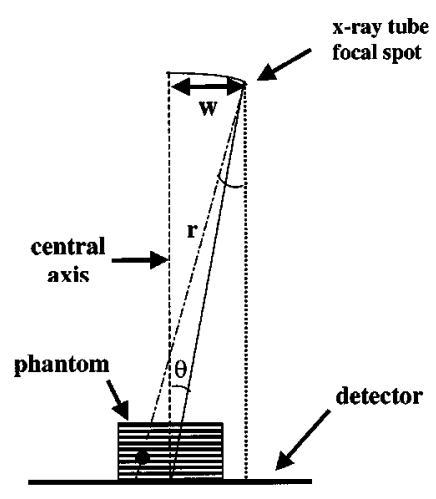

(a)

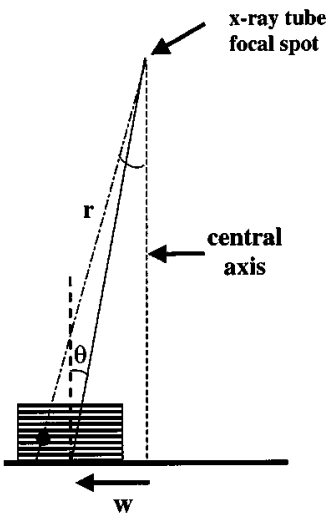

(b)
FIG. 1. Tube shift (a) and phantom shift (b) methods of stereo image acquisition. Both sketches illustrate the geometries for the generation of the righteye images. In (a) the x-ray tube focal spot shifts a distance $w$ to the right. In (b), the X-ray tube focal spot remains fixed along the central axis of the detector, and the phantom shifts a distance $w$ to the left. The sketches illustrate the equivalency of the geometries of the two methods. Notice that they ray $r$ passes through the black circular object in the phantom at the same angle in both cases. The corresponding drawing for the left-eye images of the stereo pairs would have the X-ray tube in (a) shifted to the left a distance $w$, and the phantom in (b) shifted a distance $w$ to the right.

pixel size of our previous images obtained with the Fischer MammoVision stereotaxic unit. The GE system employs a digital detector consisting of a CsI:Tl light converter and an $a$-Si active matrix flat panel unit with photodiodes and TFT pixel switches. ${ }^{15}$ The detector measures $23 \mathrm{~cm} \times 19 \mathrm{~cm}$. Stereo images are traditionally produced by shifting the $\mathrm{x}$-ray tube to the right and left of an axis perpendicular to the detector. Usually, the total x-ray tube shift is $10 \%$ of the focus-to-detector distance, which corresponds, with a stereo shift angle of about $\pm 3^{\circ}\left[=\tan ^{-1}(0.1 / 2)\right]$. For phantom imaging, an alternative to shifting the $\mathrm{x}$-ray tube is shifting the phantom. This is illustrated in Fig. 1. We employed this method for all of the contact images in this study. We built a phantom sliding device with positioning marks at the locations for the two shift angles that were studied $\left( \pm 3^{\circ}\right.$ and $\left.\pm 6^{\circ}\right)$. Note for illustration purposes, to simplify the comparison of the methods, that the x-ray tube in Fig. 1 is shown rotating about a fulcrum at the center of the detector, with a stereo shift angle, $\theta$. In the calculation of the shift distance, $w$, for our phantom-shift imaging setup, the actual geometry was used (i.e., a source-to-detector distance of $66 \mathrm{~cm}$, a fulcrum $46 \mathrm{~cm}$ from the focal spot, and a magnification-standto-detector distance of $26.4 \mathrm{~cm}$ ). The small difference in the focus-to-detector and focus-to-object distances for the tubeshift and phantom-shift methods, when the tube shift involves $\mathrm{x}$-ray tube rotation about a fulcrum, was neglected in our study. For the actual contact and magnification geometries that were employed, it can be shown that these differ- 
ences have a $0.6 \%$ or smaller effect on the accuracy of the results, which is essentially negligible (e.g., $0.6 \%$ of $1.0 \mathrm{~mm}$ accuracy $=0.006 \mathrm{~mm}$ ).

When using the tube-shift and phantom-shift methods, it is desirable to align the resulting images such that an object (e.g., a fibril) in contact with the bottom of the phantom does not shift. By doing so, all depths or distances of objects in the phantom will be measured relative to the back surface of the phantom. We achieved the desired zero shift by placing a fiducial marker on the top surface of the slider on which the phantom was placed and digitally translating the resulting left- and right-eye images so that the fiducial markers coincided. For magnification mammography, especially at larger stereo shift angles, the phantom shift method cannot be used because only a portion of the phantom will project to within the field of view of the detector due to the limited size of the detector. For our magnification techniques, we employed the phantom-shift method for the $\pm 3^{\circ}$ stereo image acquisition and the tube-shift method for the $\pm 6^{\circ}$ stereo image acquisition. All images in this study were obtained using a technique of $30 \mathrm{kVp}$, Rh filter, Rh target, $63 \mathrm{mAs}$. The large (nominal $0.3 \mathrm{~mm}$ ) focal spot was employed for the contact images and the small (nominal $0.15 \mathrm{~mm}$ ) focal spot for the $(1.8 \times)$ geometric magnification images. The scatter-rejection grid was removed for magnification image acquisition.

\section{Stereo image display}

The stereoscopic display system that was employed for this study consisted of a Barco-Metheus (Beaverton, OR) model 1760S stereo graphics board in a SUN Microsystems (Palo Alto, CA) Ultra 10 computer. The Metheus board operates in a page flipping stereoscopic mode whereby the leftand right-eye images are displayed sequentially, one after the other. This board is capable of displaying $1408 \times 1408 \times 8$ bit progressive-scan images at a refresh rate of $114 \mathrm{~Hz}$. The images in our study were displayed on a $21 \mathrm{in.} \mathrm{Barco} \mathrm{model}$ 521 monitor and viewed with NuVision (Beaverton, OR) LCD stereoscopic glasses. We employed in-house developed software to display, pan, zoom, and adjust the contrast and brightness of the images.

\section{Virtual cursor}

We developed software to generate the virtual cursors and display their $x, y$, and $z$ positions. The 3-D nature of the cursor is achieved by introducing offsets in the horizontal positions of the representations of the cursor in the left- and right-eye images. The $z$ coordinate is equal to the offset. When the offset is 0 , the cursor is at the same $x, y$ position in both images, and it appears stereoscopically to be at the depth of the monitor screen. As the horizontal offset between the cursor positions is increased in one direction (e.g., left), the cursor appears to move closer to the observer, and as the offset is increased in the opposite direction (e.g., right), the cursor appears to move toward or into the monitor.

\section{E. Z-coordinate calibration}

The $z$ coordinate was calibrated by imaging thin wires placed on the steps of a solid acrylic step wedge accurately milled with known step heights. The step wedge was imaged with the GE digital mammography system using the same phantom shift or x-ray tube shift as for the images of the fibril phantoms under the corresponding imaging conditions, and using the fiducial marker alignment technique described previously. The thin wires that were employed for calibration were oriented perpendicular to the tube shift direction. The resulting stereoscopic images were viewed without the stereo glasses and the left- and right-eye cursor positions were adjusted to overlay the left- and right-eye images of the wires on the steps. The $z$ coordinates of the cursor were linearly fit to the known depths of the wires to obtain the calibration line. This calibration was performed for each of the image magnification/zoom conditions discussed below. While this calibration method is accurate and highly reproducible, it relies on the ability of the user to match the positions of the cursors and fibrils in the image and is therefore subjective. A future improvement of the calibration method would entail developing a computer program to determine the positions of the fibrils (e.g., their centers of masses).

\section{F. True fibril depths}

Through a careful examination of the fibril phantom, we noticed that minor warping of the sandwiched Lexan plates could cause the actual depths of the fibrils to differ from their nominal 1, 3, 5, 7, 9, and $11 \mathrm{~mm}$ values. To more accurately determine the true depths of the fibrils for each phantom layer configuration, we applied the calibration method described above to one set of the stereo pair images. The $\pm 6^{\circ}$ magnification image pair was selected because the displacements in the fibril locations between the left- and right-eye images are the greatest for this image pair. The larger displacement results in greater localization accuracy since the limitation of approximately \pm 1 pixel uncertainty in placement of the stereo cursors on the fibrils in the images will correspond to a smaller uncertainty in the actual depth. A third observer who was different from the two who participated in the observer study described below viewed, without the stereo glasses, the $\pm 6^{\circ}$ magnification stereo pair images of the phantoms in the three multilayer configurations studied and adjusted the left- and right-eye cursor components to overlay each fibril. The measured $z$ values were then converted to true depths in millimeters using the calibration lines derived with the step wedge phantom. In performing this procedure, we found that when the nominal $1 \mathrm{~mm}$ depth fibrils (i.e., those that were a distance of $1 \mathrm{~mm}$ from the bottom of the phantom) were viewed without the glasses, they were too close to each other for the accurate positioning of overlaying cursors. We therefore could not determine their true depths and only analyzed the true depths of the fibrils at the nominal 3, 5, 7, 9, and $11 \mathrm{~mm}$ depths in the phantoms. Hence, the results in this paper are only presented at those depths. This is a consequence of the method that was employed to determine the true depths and would not be a limi- 
tation for a test object that was perfectly flat at each level since the true depths would then be equal to the nominal depths.

\section{G. Observer study}

For the observer experiment, we arranged the multilayered phantom in one configuration, and had two participants use the stereoscopic virtual cursor system to measure the depths of the fibrils in stereomammograms of the phantom for the seven stereo angle/geometry/display conditions described in the following: (1) $\pm 3^{\circ}$ stereo, contact geometry, no zoom; (2) $\pm 3^{\circ}$ stereo, contact geometry, zoom $=2 \times$; (3) $\pm 6^{\circ}$ stereo, contact geometry, no zoom; (4) \pm 6 stereo, contact geometry, zoom $=2 \times$; (5) $\pm 3^{\circ}$ stereo, geometric magnification $=1.8 \times$, no zoom; (6) $\pm 3^{\circ}$ stereo, geometric magnification $=1.8 \times$, zoom $=2 \times$; and (7) $\pm 6^{\circ}$ stereo, geometric magnification $=1.8 \times$, no zoom.

In addition, one of the observers repeated the entire study for two other phantom configurations. That observer also made a second set of measurements on one of the images (contact geometry with $\pm 6^{\circ}$ stereo shift angle) 8 months after the initial reading to assess reproducibility.

The stereo acuity of both observers was tested using a standard Randot ${ }^{\circledR}$ Circles Stereo test (Stereo Optical Co., Inc., Chicago, IL). In this test, the subject views a set of ten objects on the test pattern through polarized glasses. Each object consists of three circles, one of which when viewed stereoscopically should appear to be closer to the observer than the other two. The test subject is asked to identify the circle that appears closest in each object. Both observers in our study accurately identified each circle that was closer to them for all cases, indicating their level of stereopsis is at least $20 \mathrm{~s}$ of arc at a viewing distance of 16 in. Their performance is comparable to the average $(21.3 \mathrm{~s}$ of arc) that has been measured with this test pattern for adults with excellent, balanced monocular visual acuity (at least 20/20 in each eye and equal acuity in both eyes). ${ }^{16}$

The cursor that was employed in our studies was a black cross-shaped cursor. It was symmetrically shaped with an overall height of 64 pixels, and an overall width of 64 pixels. The lines were two pixels thick, and the arrowheads at each end of the lines were three pixels long. In a brief preliminary study, it was found that a cursor like this that has vertical and horizontal lines worked best for measuring the depths of the $+45^{\circ}$ and $-45^{\circ}$ oriented fibrils.

The observers recorded their measured $z$ coordinates of each fibril, and these $z$ coordinates were converted into depths (or actually distances in front of the back surface of the phantom) using calibration lines that were calculated from the step wedge data discussed above. These depths were then compared with the known depths both by performing linear least-squares fits and by computing the rms and mean errors. Parameters of linear least-squares fits that were compared for each observer for the seven stereo angle/ geometry/display zoom combinations included the slope, intercept, correlation coefficient ( $r$ value), and standard error of

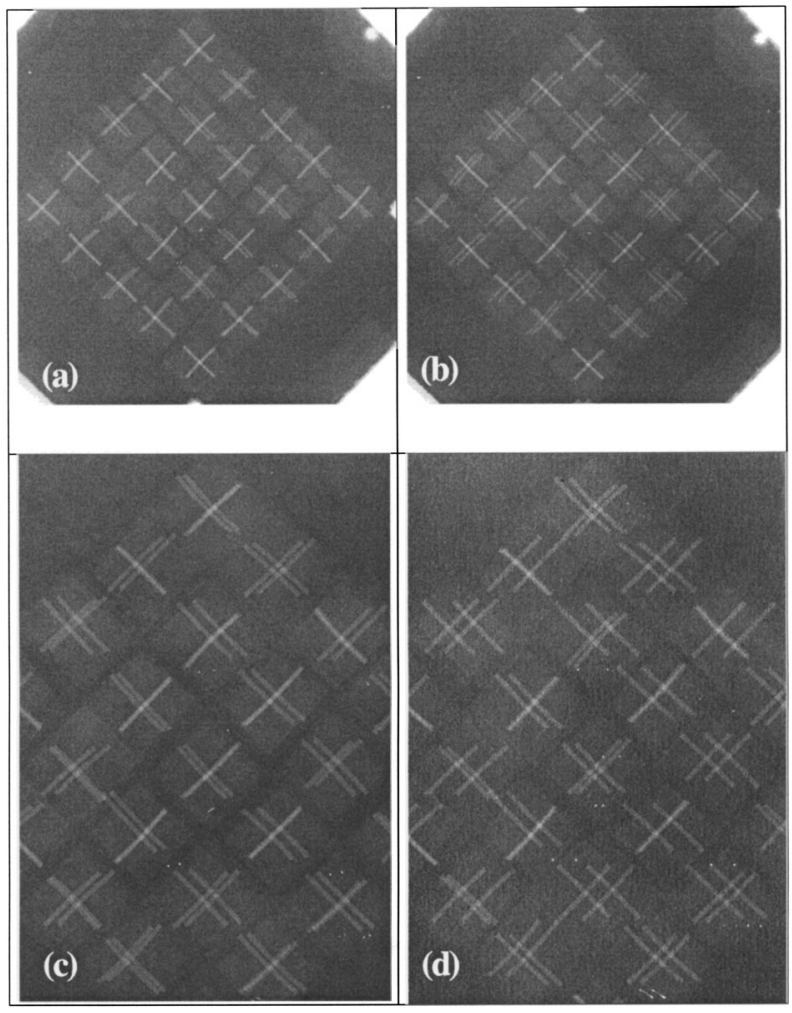

FIG. 2. Examples of images for (a) $\pm 3^{\circ}$ stereo shift angle, contact geometry, (b) $\pm 6^{\circ}$ stereo shift angle, contact geometry, (c) $\pm 3^{\circ}$ stereo shift angle magnification geometry, and (d) $\pm 6^{\circ}$ stereo shift angle magnification geometry. Note the combined images in this figure were created for illustration purposes by averaging the left- and right-eye images. In the actual stereo display, the left- and right-eye images are perceived individually. The averaging process helps illustrate the two images at once, but reduces the true image contrast.

the estimate (SEE). The rms errors were computed using the equation

$$
\text { rms error }=\sqrt{\frac{\sum_{i=1}^{N}\left(\text { true depth }_{i}-\text { measured } \text { depth }_{i}\right)^{2}}{N}},
$$

when $N$ was the total number of fibrils excluding those at the nominal $1 \mathrm{~mm}$ depth ( $N$ was equal to 40 for one of the phantom configurations and 42 for the other two phantom configurations). Finally, two-tailed paired $t$ tests of the differences between the measured and true $z$ coordinates of the fibrils for the various stereo imaging/viewing techniques were performed to determine the statistical significance of those differences.

\section{RESULTS}

Combined left-eye and right-eye images of the same phantom obtained with the following geometries: $\pm 3^{\circ}$ stereo shift—contact, $\pm 6^{\circ}$ stereo shift—contact, $\pm 3^{\circ}$ stereo shiftmagnification, and $\pm 6^{\circ}$ stereo shift-magnification are shown in Fig. 2. Examples of the calibration lines that were computed from the step wedge measurements are shown in Fig. 3. The calibration lines for the $\pm 3^{\circ}$ and $\pm 6^{\circ}$ stereo shift angles in contact geometry are compared in part (a) of this figure, and the corresponding lines for the magnification ge- 

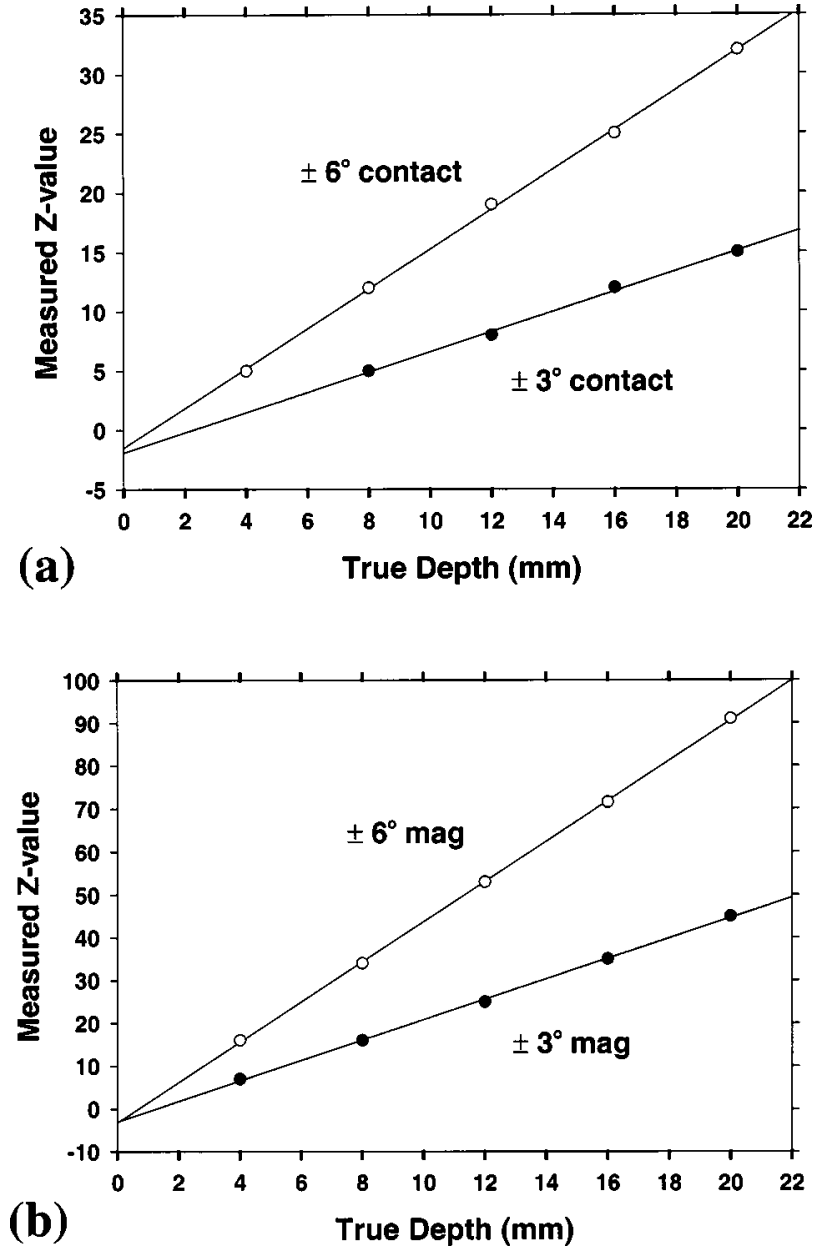

Fig. 3. (a) Calibration lines for $\pm 3^{\circ}$ stereo shift contact and $\pm 6^{\circ}$ stereo shift contact acquisition; (b) calibration lines for $\pm 3^{\circ}$ stereo shift magnification and $\pm 6^{\circ}$ stereo shift magnification acquisition.

ometry are compared in part (b). For the $2 \times$-zoom mode, our cursor software effectively incremented the $z$ values in steps of 0.5 per pixel shift instead of steps of 1 for the nonzoom mode. Consequently, the calibration lines that were computed in the $2 \times$-zoom display modes were nearly identical to those in the nonzoom modes and they are therefore not shown in the figures. The calibration equations for the conversion of the measured $z$ values to the depths (distances from the back of the phantoms) for the various imaging conditions are listed in Table I, below.
TABLE I. Calibration equations for converting measured $z$ values to depths (distances in front of the backside of the phantom). Note: these calibration equations were derived by linearly fitting the data acquired from the step wedge images. Examples of the lines for the step wedge data are shown in Fig. 1.

\begin{tabular}{cccc}
\hline $\begin{array}{c}\text { Stereo Shift } \\
\text { Angle }\end{array}$ & Geometry & Display zoom & Equation \\
\hline \pm 3 degrees & Contact & None & Depth $(\mathrm{mm})=\frac{Z+1.9}{0.85}$ \\
\pm 3 degrees & Contact & $2 \times$ & Depth $(\mathrm{mm})=\frac{Z+1.55}{0.825}$ \\
\pm 6 degrees & Contact & None & Depth $(\mathrm{mm})=\frac{Z+1.5}{1.675}$ \\
\pm 6 degrees & Contact & $2 \times$ & Depty $(\mathrm{mm})=\frac{Z+2.5}{1.75}$ \\
\pm 3 degrees & Magnification & None & Depth $(\mathrm{mm})=\frac{Z+2.9}{2.375}$ \\
\pm 3 degrees & Magnification & $2 \times$ & Depth $(\mathrm{mm})=\frac{Z+2.6}{2.35}$ \\
\pm 6 degrees & Magnification & None & Depth $(\mathrm{mm})=\frac{Z+3.15}{4.688}$ \\
\hline \hline
\end{tabular}

Results comparing the performances of the two observers for the various stereo angle, geometric magnification, and display zoom combinations of the experiment are summarized in Tables II-IV. Examples of plots of the measured versus true depths for one observer for each of the imaging conditions are shown in Fig. 4. Errors in the depth measurements for each imaging condition for the one observer who viewed images in two additional phantom configurations are listed in Table IV. The paired $t$-test results for the observer who measured the $z$ values of the fibrils in the phantom in three separate phantom layer configurations are listed in Table V. (Note, the paired $t$-test results for the other observer who made measurements of the fibrils in the first phantom configuration were very similar to those listed in part A of this table and are therefore not shown.)

The reproducibility of the observer's measurements in the same image read twice was excellent. For the 40 fibrils that were analyzed in the image, 35 of the measured $z$ values were the same for both readings, and the remaining $5 z$ values differed by \pm 1 . This translates to a rms difference in the $z$ values of 0.354 (i.e., $\sqrt{5 / 40}$ ), which is equal to $0.21 \mathrm{~mm}$.

TABLE II. Linear fit parameters for measured versus true depths of fibrils for two observers (values for observer 1 are indicated by subscript 1 , and for observer 2 by subscript 2 ).

\begin{tabular}{lccrrrrr}
\hline \hline & $3^{\circ}$ & $3^{\circ}$ zoom & \multicolumn{1}{c}{$6^{\circ}$} & $6^{\circ}$ zoom & $3^{\circ}$ mag & $3^{\circ}$ mag zoom & $6^{\circ}$ mag \\
\hline$r_{1}$ & 0.956 & 0.949 & 0.994 & 0.996 & 0.982 & 0.978 & 0.998 \\
$r_{2}$ & 0.934 & 0.921 & 0.994 & 0.996 & 0.985 & 0.981 & 0.998 \\
intercept $_{1}$ & 0.669 & 0.030 & -0.757 & -0.306 & -0.822 & -0.470 & -0.013 \\
intercept $_{2}$ & 1.379 & 0.930 & -0.910 & -0.502 & -0.777 & -0.476 & -0.082 \\
slope $_{1}$ & 0.969 & 1.041 & 1.000 & 0.978 & 1.031 & 1.007 & 0.994 \\
slope $_{2}$ & 0.961 & 1.002 & 1.037 & 0.997 & 1.049 & 1.016 & 1.004 \\
SEE $_{1}$ & 0.884 & 1.036 & 0.331 & 0.263 & 0.604 & 0.656 & 0.170 \\
$\mathrm{SEE}_{3}$ & 1.103 & 1.264 & 0.329 & 0.254 & 0.560 & 0.606 & 0.174 \\
\hline \hline
\end{tabular}


TABLE III. Root mean square (rms), mean, and standard deviations of depth errors in mm (values for observer 1 are indicated by subscript 1 , and for observer 2 by subscript 2 ).

\begin{tabular}{lccrrrrr}
\hline \hline & $3^{\circ}$ & $3^{\circ}$ zoom & $6^{\circ}$ & $6^{\circ}$ zoom & $3^{\circ}$ mag & $3^{\circ}$ mag zoom & $6^{\circ}$ mag \\
\hline RMS $_{1}$ & 0.963 & 1.077 & 0.820 & 0.550 & 0.839 & 0.766 & 0.179 \\
RMS $_{2}$ & 1.520 & 1.553 & 0.706 & 0.582 & 0.702 & 0.692 & 0.179 \\
Mean error $_{1}$ & 0.420 & 0.354 & -0.754 & -0.482 & -0.591 & -0.421 & -0.064 \\
Mean error & 1.068 & 0.945 & -0.619 & -0.527 & -0.417 & -0.357 & -0.053 \\
Std dev & 0.877 & 1.030 & 0.326 & 0.268 & 0.603 & 0.648 & 0.169 \\
Std dev & 1.095 & 1.247 & 0.343 & 0.251 & 0.571 & 0.600 & 0.173 \\
\hline \hline
\end{tabular}

The computed rms errors (relative to the true depths) for the two independent readings were essentially identical $(0.706$ $\mathrm{mm})$.

\section{DISCUSSION}

The average rms errors for the two observers reading the same sets of images (Table III) were $1.2 \mathrm{~mm}\left( \pm 3^{\circ}\right.$ contact, no zoom), $1.3 \mathrm{~mm}\left( \pm 3^{\circ}\right.$ contact, zoom), $0.8 \mathrm{~mm}\left( \pm 6^{\circ}\right.$ contact, no zoom), $0.6 \mathrm{~mm}$ ( $\pm 6^{\circ}$ contact, zoom), $0.8 \mathrm{~mm}\left( \pm 3^{\circ}\right.$ magnification, no zoom), $0.7 \mathrm{~mm}\left( \pm 3^{\circ}\right.$ magnification, zoom), and $0.2 \mathrm{~mm}$ ( $\pm 6^{\circ}$ magnification, no zoom). Corresponding values for the one observer who viewed all images of the phantom in three different layer configurations (Tables III and IV) were 1.6, 1.5, 0.7, 0.7, 0.5, 0.6, and $0.2 \mathrm{~mm}$, respectively. In general, better results were obtained for the larger shift angle and for magnification geometry. Both of these improvements are in agreement with the analysis by Jiang et al. of the theoretical trends for stereo localization accuracy. ${ }^{17}$ According to Jiang et al., depth (z) localization error is inversely proportional to the x-ray tube shift (which is approximately equal to the tangent of the shift angle in our case), and directly proportional to the square of the focal spot-to-object distance (i.e., the error is smaller when the object is closer to the focal spot as in magnification geometry). Jiang et al. also showed that the sensitivity of the measurements to small changes in depth is proportional to the tube shift, and inversely proportional to the square of the focus-to-object distance.

Combining both observers' experimental results for all contact geometry images, we found that increasing the stereo shift angle from the conventional $\pm 3^{\circ}$ to $\pm 6^{\circ}$ improved the depth measurement accuracy by factors of about 1.2 to 4.0. The theoretical improvement considering only geometric factors ${ }^{17}$ is approximately equal to the ratio of the tangents of the stereo shift angles which is 2.0 for this case $\left(=\tan 6^{\circ} \%\right.$ $\tan 3^{\circ}$ ).

The variability in our results may be due to other sources of error. These include errors in the x-ray tube and slider positions for the acquisition of both the test phantom and calibration step wedge images, errors in the matching of the fiducial marker positions in the images for the desired 0 displacement at the bottom of the phantom, errors due to the limitation of a minimum 1 pixel (as opposed to fractional pixel) increment in the positioning of the virtual cursor for the $z$-value measurements in the test phantom and calibration phantom images, and uncertainties in the readers' determination of the cursor depth to overlay the fibril. The error in the measured depth due to the 1-pixel increment in the virtual cursor position is a function of the imaging geometry and can be computed by taking the derivative of the slope of the calibration line (Table I). This error is $1.18 \mathrm{~mm}$ for the $\pm 3^{\circ}$ stereo shift-contact geometry and $0.60 \mathrm{~mm}$ for the $\pm 6^{\circ}$ stereo shift-contact geometry. Thus, a change of $1 z$-value unit in an observer's depth measurement of a fibril has a much greater effect for $\pm 3^{\circ}$ contact than for $6^{\circ}$ contact geometry.

Comparing all measurements in the magnification versus contact geometries, we found improvements in depth accuracy by factors of $1.1-10.2$ for $\pm 3^{\circ}$ magnification versus $\pm 3^{\circ}$ contact and by factors of 3.0-4.6 for $\pm 6^{\circ}$ magnification versus $\pm 6^{\circ}$ contact. The theoretical improvement due to geometrical factors in both cases is approximately proportional to the square of the ratio of the focus-to-object distances. ${ }^{17}$ Considering a fibril located at about the midplane of the phantom ( $5 \mathrm{~mm}$ from the bottom of the phantom), and using the actual focus-to-magnification stand distance of $39.6 \mathrm{~cm}$, and the focus-to-detector distance of $66 \mathrm{~cm}$ (assuming, for simplicity, that in the contact mode, the phantom is directly

TABLE IV. Root mean square (rms), mean, and standard deviations of depth errors in mm for two additional images read by observer 2 .

\begin{tabular}{|c|c|c|c|c|c|c|c|}
\hline & $3^{\circ}$ & $3^{\circ} \mathrm{zoom}$ & $6^{\circ}$ & $6^{\circ} \mathrm{zoom}$ & $3^{\circ} \mathrm{mag}$ & $3^{\circ} \mathrm{mag}$ zoom & $6^{\circ} \mathrm{mag}$ \\
\hline $\mathrm{RMS}_{\text {image } 2}$ & 0.813 & 0.572 & 0.632 & 0.782 & 0.641 & 0.763 & 0.174 \\
\hline $\mathrm{RMS}_{\text {image } 3}$ & 2.483 & 2.422 & 0.624 & 0.854 & 0.244 & 0.233 & 0.189 \\
\hline Mean error $_{\text {image } 2}$ & 0.691 & 0.303 & 0.373 & 0.620 & -0.218 & -0.271 & 0.031 \\
\hline Mean error image $3_{3}$ & 2.239 & 2.045 & 0.567 & 0.829 & 0.057 & 0.027 & 0.086 \\
\hline Standard dev $v_{\text {image } 2}$ & 0.433 & 0.490 & 0.515 & 0.482 & 0.610 & 0.722 & 0.174 \\
\hline Standard dev $v_{\text {image } 3}$ & 1.086 & 1.314 & 0.263 & 0.208 & 0.240 & 0.234 & 0.170 \\
\hline
\end{tabular}



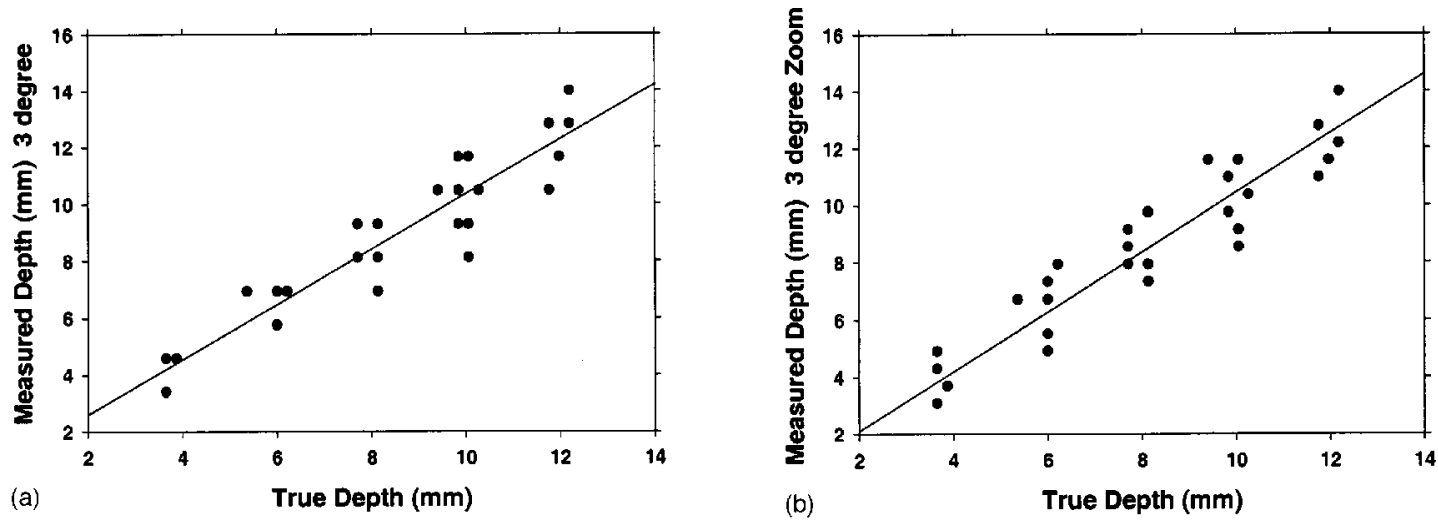

(a)

True Depth (mm)

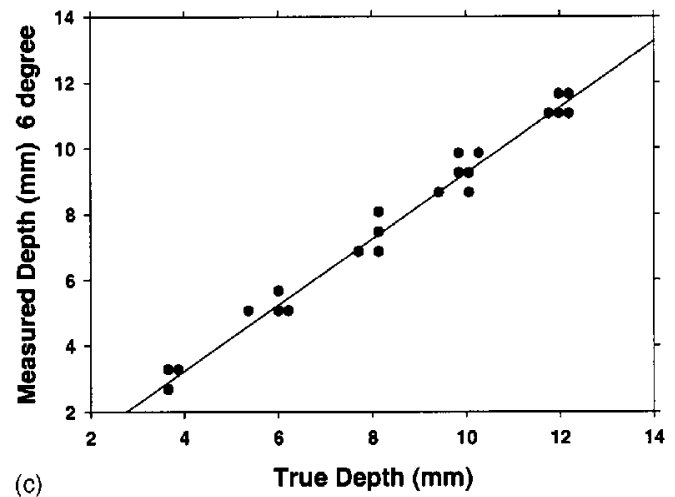

(c)

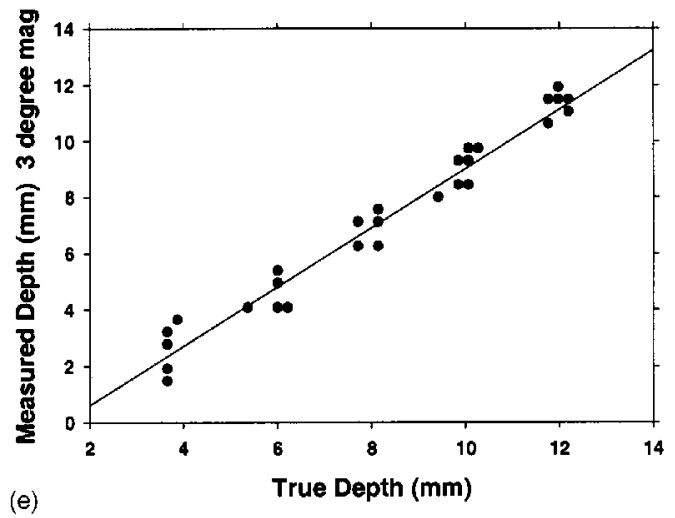

(b)

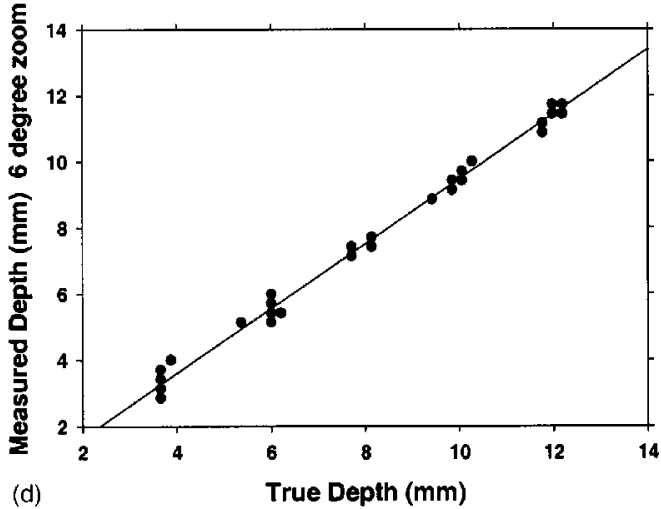

(d)

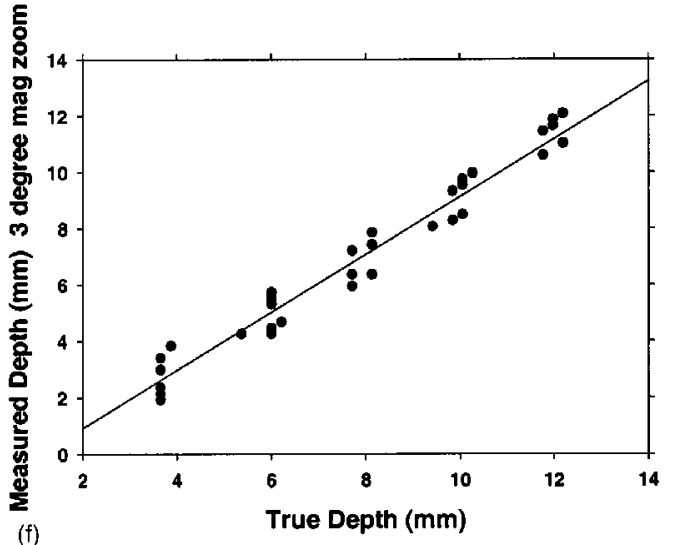

(f)

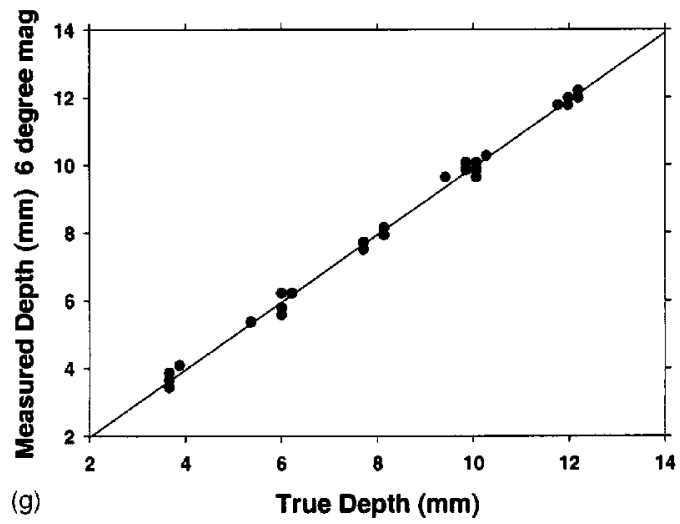

FIG. 4. Examples of measured versus true depths (distances from the back of the phantom) for observer number $1 .(\mathrm{a})$ Stereo shift angle $= \pm 3^{\circ}$, contact, no zoom; (b) stereo shift angle $= \pm 3^{\circ}$, contact, zoom $=2$; (c) stereo shift angle $= \pm 6^{\circ}$, contact, no zoom; (d) stereo shift angle $= \pm 6^{\circ}$, contact, zoom $=2$; (e) stereo shift angle $= \pm 3^{\circ}$, magnification mode, no zoom; (f) stereo shift angle $= \pm 3^{\circ}$, magnification mode, zoom $=2 ;(\mathrm{g})$ stereo shift angle $= \pm 6^{\circ}$, magnification mode, no zoom. 
TABLE V. $p$ values for the paired $t$ tests comparing the differences between the observed and measured $z$ coordinates of the fibrils for the various stereoscopic imaging and display techniques (values are for three phantom configurations (different fibril patterns) analyzed by observer $2(*=$ not significant since $p>0.05$; the rest are significant; note: the value of 0.00000 means $p<10^{-5}$ ).

\begin{tabular}{|c|c|c|c|c|c|c|}
\hline A Phantom & $\begin{array}{l}\text { configuration } \\
3^{\circ} \text { contact } \\
\text { zoom }\end{array}$ & $6^{\circ}$ contact & $\begin{array}{l}6^{\circ} \text { contact } \\
\text { zoom }\end{array}$ & $3^{\circ} \mathrm{mag}$ & $\begin{array}{c}3^{\circ} \mathrm{mag} \\
\text { zoom }\end{array}$ & $6^{\circ} \mathrm{mag}$ \\
\hline $3^{\circ}$ contact & $0.22191 * a$ & 0.00000 & 0.00000 & 0.00000 & 0.00000 & 0.00000 \\
\hline $\begin{array}{l}3^{\circ} \text { contact } \\
\text { zoom }\end{array}$ & & 0.00000 & 0.00000 & 0.00002 & 0.00005 & 0.00001 \\
\hline $6^{\circ}$ contact & & & 0.04878 & $0.03859 \mathbf{a}$ & 0.01282 & 0.00000 \\
\hline $\begin{array}{l}6^{\circ} \text { contact } \\
\text { zoom }\end{array}$ & & & & $0.27275^{*} \mathrm{a}$ & $0.10784 * a$ & 0.00000 \\
\hline $3^{\circ} \mathrm{mag}$ & & & & & 0.03496 & 0.00039 \\
\hline $3^{\circ} \mathrm{mag}$ & & & & & & 0.00416 \\
\hline
\end{tabular}

a indicates not significant $(p>0.05)$ for observer 1

B. Phantom layer configuration \#2

\begin{tabular}{|c|c|c|c|c|c|c|}
\hline & $\begin{array}{c}3^{\circ} \text { contact } \\
\text { zoom }\end{array}$ & $6^{\circ}$ contact & $\begin{array}{c}6^{\circ} \text { contact } \\
\text { zoom }\end{array}$ & $3^{\circ} \mathrm{mag}$ & $\begin{array}{c}3^{\circ} \mathrm{mag} \\
\text { zoom }\end{array}$ & $6^{\circ} \mathrm{mag}$ \\
\hline $3^{\circ}$ contact & 0.00000 & 0.00123 & $0.46220^{*}$ & 0.00000 & 0.00000 & 0.00000 \\
\hline \multicolumn{6}{|l|}{ zoom } & 0.00152 \\
\hline $6^{\circ}$ contact & & & 0.00000 & 0.00031 & 0.00045 & 0.00012 \\
\hline $6^{\circ}$ contact & & & & 0.00000 & 0.00000 & 0.00000 \\
\hline \multicolumn{7}{|l|}{ zoom } \\
\hline $3^{\circ} \mathrm{mag}$ & & & & & $1.14830^{*}$ & 0.00541 \\
\hline $3^{\circ} \mathrm{mag}$ & & & & & & 0.00538 \\
\hline \multicolumn{7}{|l|}{ zoom } \\
\hline \multicolumn{7}{|c|}{ C. Phantom layer configuration \#3 } \\
\hline & $\begin{array}{c}3^{\circ} \text { contact } \\
\text { zoom }\end{array}$ & $6^{\circ}$ contact & $\begin{array}{c}6^{\circ} \text { contact } \\
\text { zoom }\end{array}$ & $3^{\circ} \mathrm{mag}$ & $\begin{array}{l}3^{\circ} \mathrm{mag} \\
\text { zoom }\end{array}$ & $6^{\circ} \mathrm{mag}$ \\
\hline $3^{\circ}$ contact & 0.01394 & 0.00000 & 0.00000 & 0.00000 & 0.00000 & 0.00000 \\
\hline $\begin{array}{l}3^{\circ} \text { contact } \\
\text { zoom }\end{array}$ & & 0.00000 & 0.00000 & 0.00000 & 0.00000 & 0.00000 \\
\hline $6^{\circ}$ contact & & & 0.00000 & 0.00000 & 0.00000 & 0.00000 \\
\hline $6^{\circ}$ contact & & & & 0.00000 & 0.00000 & 0.00000 \\
\hline \multicolumn{7}{|l|}{ zoom } \\
\hline $3^{\circ} \mathrm{mag}$ & & & & & $0.25839 *$ & $0.45679 *$ \\
\hline $3^{\circ} \mathrm{mag}$ & & & & & & $0.11153 *$ \\
\hline zoom & & & & & & \\
\hline
\end{tabular}

on top of the detector, and the fulcrum is in the plane of the detector), the theoretical improvement in depth accuracy is about a factor of 2.8. Variations in the observed improvements can be attributed to the reasons listed above.

In three of four cases, the measurement accuracies for $\pm 3^{\circ}$ magnification geometry were nearly identical to those for $\pm 6^{\circ}$ contact geometry. In one case, the accuracy was significantly superior $(0.24 \mathrm{~mm}$ vs $0.62 \mathrm{~mm})$ in \pm 3 magnification as compared to $\pm 6^{\circ}$ contact. The theoretical improvements due to geometrical considerations for this comparison is about 1.4. (As discussed above, the $\pm 6^{\circ}$ contact results should be better than the $\pm 3^{\circ}$ contact by a factor of 2 due to the increased tube shift, and the $\pm 3^{\circ}$ magnification results should be better than the $\pm 3^{\circ}$ contact by a factor of 2.8 due to the reduced focus-to-object distance. Combining these factors, the $\pm 3^{\circ}$ magnification measurements should be more accurate than $\pm 6^{\circ}$ contact by a factor of $1.4(=2.8 / 2)$.
The errors due to one-unit variations in the measured $z$ values for the two geometries are similar $\left(0.6 \mathrm{~mm}\right.$ for $\pm 6^{\circ}$ contact and $0.42 \mathrm{~mm}$ for $\pm 3^{\circ}$ magnification.) Considering these factors and the other sources of measurement error discussed previously, the lack of observed improvement for the $\pm 3^{\circ}$ magnification geometry as compared to the $\pm 6^{\circ}$ contact geometry in most cases is not surprising. It should be noted that the rms errors for both geometries are very small (less than $1 \mathrm{~mm}$ ) so either geometry would be adequate for making depth measurements.

Zooming the stereo images by a factor of 2 did not seem to improve the depth measurement accuracy. Although sometimes it appeared to improve the accuracy (7 of 12 times), other times it reduced the accuracy (5 of 12 times). There was basically no improvement on average (the difference in the average rms error for no zoom versus zoom, was 0.14 $\mathrm{mm})$. The small differences one way or the other were there- 
fore likely caused by experimental uncertainties due to the many factors described above. Thus, artificially increasing the displacement between objects viewed in the left- and right-eye stereo pairs through zooming the display does not have the same effect as increasing the displacement via increasing the stereo shift angle or increasing the geometric magnification. The stereo effect was more readily visualized in the zoom mode, but the signal-to-noise ratios of the fibril images were basically the same as those in the images displayed without zoom. In contrast, the acquisition of images with geometric magnification actually improves the signalto-noise ratio. ${ }^{18}$ The $2 \times$ zoom that was employed in this study was achieved by pixel replication. Results may be different for interpolative zoom. Finally, the use of greater zoom factors was also not explored. However, based on the results of a recently published study, increasing the display zoom factor may not be beneficial. ${ }^{19}$ In that study, the observers' stereoacuities and depth perceptions were compared using a standard Randot stereotest pattern, with and without magnification via a $4 \times$ optical loupe and a $16 \times$ microscope. The researchers found that stereo acuity and depth perception decreased with increasing optical magnification of the pattern.

The paired $t$-test results listed in Table $\mathrm{V}$ indicate the majority of the differences between the depth measurement accuracies obtained with the $\pm 3^{\circ}$ and $\pm 6^{\circ}$ stereo shift angles, magnification and contact geometries, and normal and zoom displays are statistically significant $(p<0.05)$. It is interesting to note that there was little consistency between the categories of the small number of insignificant differences $(p$ $>0.05$ ) for the images created with the three different phantom configurations (parts A, B, and C of Table V). The only consistent insignificant result was that in two of the three cases, the accuracies for the $\pm 3^{\circ} \mathrm{mag}$ and $\pm 3^{\circ} \mathrm{mag}$ zoom depth measurements were not statistically significant. The $t$-test results for the two observers were quite similar for phantom configuration \#1 (see footnote "a" of Table V, part A). Increasing the number of observers would have increased the statistical power of this study; however, the variability in the results due to the various factors described above would not have been reduced. Therefore the conclusions would likely be the same.

The best accuracy of about $0.2 \mathrm{~mm}$, among all cases, was obtained with geometric magnification using a stereo angle of $\pm 6^{\circ}$. Therefore, this is the mode we recommend for obtaining accurate depth measurements with virtual cursors in stereomammograms. Conventional stereoradiography is performed in contact mode using a stereo shift angle of $\pm 3^{\circ}$. According to Christensen, ${ }^{20}$ this angle was determined empirically "by trial and error." The angle is a compromise between the improved stereoscopic effect with increasing angle, the increased eye strain and difficulty in fusing the left- and right-eye images, especially at larger stereo angles, and the reduced patient coverage at larger stereo angles. For our particular case, the increased stereoscopic effect associated with the use of twice the conventional stereo angle and the use of $\sim 1.8 \times$ geometric magnification did not cause undue eye strain. It did reduce the imaged field of view and this geometry could not be used for imaging an entire breast unless a larger-area detector was employed. Based on our measurements and observations, we recommend for stereoscopic imaging and depth measurements within an entire breast with the GE full-field digital mammography system, that a contact geometry be employed using a stereoshift angle of $\pm 6^{\circ}$ instead of the conventional $\pm 3^{\circ}$. The overall results and recommendations of this study may differ for detectors having different pixel sizes and noise properties and for displays with different noise and contrast. A further investigation of the effects of these factors is warranted.

Finally, the depth measurement accuracies of the two observers in this study (Table III) were almost identical (in nearly all cases they were within $0.1 \mathrm{~mm}$ of each other). Both observers had excellent stereo acuity, and it would be expected that others with similar stereo acuity could achieve similar results after a period of training. Our previous studies with other observers have shown that there is a wide range of accuracies for depth measurements, especially for horizontally oriented objects. ${ }^{3}$ All of the fibrils in this study had horizontal and vertical components, so it would be expected that even observers who have difficulties measuring the depths of horizontally oriented objects would be able to measure the depths of the diagonally oriented fibrils, although their accuracies may not be as high. Since it is unlikely that fibrous tissues or spicules from masses in mammograms are exactly horizontal to the stereo shift direction, the angulation would enable reasonably accurate measurements in clinical images. However, the inhomogeneous anatomical background within the clinical images may partially obscure the fibrils, which would increase the difficulty of making accurate depth measurements with the stereo cursor. It is possible that with additional training and the use of depth cues (e.g., 3-D wire boxes placed about the objects of interest, or 3-D rulers in the image) in clinical images, the depth measurement accuracies of most viewers would be adequate. The development of such depth cues will be the subject of our future investigations.

\section{ACKNOWLEDGMENTS}

This work is supported by U.S. Army Medical Research and Material Command Grants No. DAMD 17-98-1-8210 and No. DAMD 17-99-1-9294. The content of this publication does not necessarily reflect the position of the funding agency, and no official endorsement of any equipment and product of any companies mentioned in this publication should be inferred.

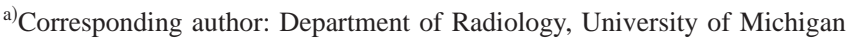
Hospitals, Room B1 F510C, 1500 East Medical Center Drive, Ann Arbor, Michigan 48109-0030. Office 734-936-7474; Fax: 734-936-7948; Electronic mail: goodsitt@umich.edu

${ }^{1}$ D. M. Chelberg, J. Hsu, C. G. Babbs, Z. Pizlo, and E. J. Delp, "Digital stereomammography," in Proceedings of the 2nd International Workshop on Digital Mammography, Excepta Medica International Congress Series 1069 (Elsevier Science, York, England, 1994), pp. 181-190.

${ }^{2}$ J. Hsu, D. M. Chelberg, C. F. Babbs, Z. Pizlo, and E. J. Delp, "Preclinical ROC studies of digital stereomammography," IEEE Trans. Med. Imaging 14, 318-327 (1995).
} 
${ }^{3}$ M. M. Goodsitt, H.P. Chan, and L. M. Hadjiiski, "Stereomammography: Evaluation of depth perception using a virtual 3D cursor," Med. Phys. 27, 1305-1310 (2000).

${ }^{4}$ D. J. Getty, R. M. Pickett, and D. J. D’Orsi, "Stereoscopic digital mammography: improving detection and diagnosis of breast cancer," in Computer Assisted Radiology and Surgery 2001, Proceedings of the 15th International Congress and Exhibition, Berlin, 27-30 June 2001, edited by H. U. Lemke, M. W. Vannier, K. Inamura, A. G. Farman, and D. Doi (Elsevier, Amsterdam, 2001; International Congress Series 1230), pp. 506-511.

${ }^{5}$ M. M. Goodsitt, H. P. Chan, J. M. Sullivan, K. L. Darner, and L. M. Hadjiiski, "Evaluation of the effect of virtual cursor shape on depth measurements in digital stereomammograms," in IWDM 2000 5th International Workshop on Digital Mammography, edited by M. J. Yaffe (Medical Physica Publishing, Madison, 2001), pp. 45-50.

${ }^{6}$ H. P. Chan, M. M. Goodsitt, K. L. Darner, J. M. Sullivan, L. M. Hadjiiski, N. Petrick, and B. Sahiner, "Effects of stereoscopic imaging technique on depth discrimination," in Ref. 5, p. 13-18.

${ }^{7}$ B. Leduc, R. Shumak, G. E. Mawdsley, M. J. Yaffe, and R. A. Jong, "Merits of using a 3-D visualization technique to target microcalcifications," in Ref. 5, pp. 56-64.

${ }^{8}$ L. T. Niklason et al., "Digital tomosynthesis in breast imaging," Radiology 205, 399-406 (1997).

${ }^{9}$ R. L. Webber, H R. Underhill, and R. I. Freimanis, "A controlled evaluation of tuned-aperture computed tomography applied to digital spot mammography," J. Digit. Imaging 13, 90-97 (2000).

${ }^{10}$ S. Suryanarayanan, A. Karellas, S. Vedantham, S. P. Baker, S. J. Glick, C. J. D'Orsi, and R. L. Webber, "Evaluation of linear and nonlinear tomosynthetic reconstruction methods in digital mammography," Acad. Radiol. 8, 291-224 (2001).
${ }^{11}$ V. Raptopoulos, J. K. Baum, M. Hochman, A. Karellas, M. J. Houlihan, and C. J. D'Orsi, "High resolution CT mammography of surgical biopsy specimens," J. Comput. Assist. Tomogr. 20, 179-184 (1996).

${ }^{12}$ J. M. Boone, T. R. Nelson, K. K. Lindfors, and J. A. Seibert, "Dedicated breast CT: Radiation dose and image quality evaluation," Radiology $\mathbf{2 2 1}$, 657-667 (2001).

13 S. L. Warren, "Roentgenologic study of the breast," Am. J. Roentgenol. 24, 113-124 (1930).

${ }^{14}$ A. D. A. Maidment, P. Bakic, and M. Alberg, "Is stereomammography possible without increasing dose?," The 6th International Workshop on Digital Mammography, 22-25 June 2002, Bremen, Germany, Program \& Abstracts, 2002, p. 27.

${ }^{15}$ S. Vedantham, A. Karellas, S. Suryanarayanan, D. Albagli, S. Han, E. J. Tkaczyk, C. E. Landberg, B. Opsahl-Ong, P. R. Granfors, I. Levis, C. J. D'Orsi, and R. E. Hendrick, "Full breast digital mammography with an amorphous silicon-based flat-panel detector: physical characteristics of a clinical prototype," Med. Phys. 27, 558-567 (2000).

${ }^{16}$ K. Simons, "Stereoacuity norms in young children," Arch. Opthalmol. 99, 439-445 (1981).

${ }^{17}$ H. Jiang, H. Liu, G. Wang, W. Chen, and L. L. Fajardo, "A localization algorithm and error analysis for stereo x-ray image guidance," Med. Phys. 27, 885-893 (2000).

${ }^{18}$ K. Doi and H. Imhof, "Noise reduction by radiographic magnification," Radiology 122, 479-487 (1977).

${ }^{19}$ L. T. Du, L. F. Wessels, J. P. Underdahl, and J. D. Auran, "Stereoacuity and depth perception decrease with increased instrument magnification: comparing non-magnified system with lens loupes and a surgical microscope," Binocul. Vis. Strabismus. Q. 16, 61-67 (2001).

${ }^{20}$ T. S. Curry, J. E. Dowdey, and R. C. Murry, Christensen's Physics of Diagnostic Radiology, 4th ed. (Lea \& Febiger, Philadelphia, 1990). 